\title{
Demographic and diagnostic profiles of older people with intellectual disability and prescription of antipsychotics
}

\author{
Anna Axmon ${ }^{1}$ (D) . Gerd Ahlström ${ }^{2}$ (D) $\cdot$ Rebecca Gagnemo Persson $^{2}$ (D) - Jonas Eberhard ${ }^{3}$ (D)
}

Received: 17 December 2018 / Accepted: 9 March 2019 / Published online: 22 March 2019

(c) The Author(s) 2019

\begin{abstract}
Purpose To describe demographic and diagnostic profiles in a national cohort of older people with intellectual disability (ID) who were prescribed antipsychotics.

Methods Using national registers, we identified people with ID who were 55 + years in $2012(n=7936)$, as well as a subcohort with complete information on demographic factors (sex, year of birth, severity of ID, presence of behavior impairment, and residence in special housing; $n=1151$ ). Data regarding diagnoses and prescription of antipsychotics were added for the time period 2006-2012. The potential effects of demographic factors and diagnoses on the prescription of sedating and lesssedating antipsychotics, respectively, were assessed in separate models by estimating the relative risks (RRs) of prescription. Results Of the demographic factors, severe/profound ID (RR 1.17), behavior impairment (RR 1.34), and living in special housing (RR 1.25) were associated with prescription of sedating antipsychotics, whereas only behavior impairment (RR 1.42) was associated with prescription of less-sedating antipsychotics. For both sedating and less-sedating antipsychotics, the diagnoses with the largest association (i.e., highest relative risk) were schizophrenia (RR 2.17 for sedating and RR 1.81 for less-sedating) and ID (RR 1.84 and RR 1.68, respectively), followed by disorders of psychological development (for sedating antipsychotics, RR 1.57) and organic mental disorders (for less-sedating antipsychotics, RR 1.55).

Conclusions The associations between prescription of antipsychotics and demographic factors and non-psychotic diagnoses, respectively, suggest that older people with ID may be prescribed antipsychotic medication without thorough psychiatric diagnosing. If so, there is a need for improving the abilities of health care professionals to properly diagnose and manage psychiatric illness in this population.
\end{abstract}

Keywords Drug prescription $\cdot$ Mental retardation $\cdot$ Aging $\cdot$ Challenging behavior $\cdot$ Schizophrenia $\cdot$ Comorbidities

\section{Introduction}

Among people with intellectual disability (ID), as well as in other populations, antipsychotic use is associated with a range of adverse events [1-4]. However, adverse events among people with ID may be difficult to identify as they are overlapping with disorders already common among

Anna Axmon

anna.axmon@med.lu.se

$1 \quad$ EPI@LUND, Division of Occupational and Environmental Medicine, Department of Laboratory Medicine, Lund University, 22180 Lund, Sweden

2 Department of Health Sciences, Faculty of Medicine, Lund University, 22100 Lund, Sweden

3 Division of Psychiatry, Department of Clinical Sciences Lund, Lund University, 25187 Helsingborg, Sweden people with ID, such as cognitive decline $[1,3,5]$. Therefore, it is essential that the prescription of antipsychotics to people with ID is made on proper indications. Several of the diagnoses considered to be indicators for prescription of antipsychotics are common among people with ID, e.g., schizophrenia [6] and anxiety [7]. Thus, a high prescription of antipsychotics among people with ID is only to be expected. Nevertheless, current prescription rates are exceeding the proportion of recorded mental illness [8]. A possible explanation for this is that antipsychotics are being used off-label to treat challenging behavior among people with ID [9-12], despite a lack of evidence of efficacy [13] as well as cost-effectiveness [14].

To determine if the number of people with ID who are prescribed antipsychotics is a cause for concern, knowledge is needed about the characteristics of people with ID and such prescriptions. The aim of the present study 
was to describe demographic and diagnostic profiles in a national cohort of older people with ID who were prescribed antipsychotics.

\section{Methods}

This is a register-based study, using Swedish national registers to define the study cohort as well as assess the outcomes investigated. Unless otherwise mentioned, data were collected for the period 2006-2012. The period was defined by availability of data regarding dispensed prescriptions.

\section{Registers}

All registers presented below are maintained by the Swedish National Board of Health and Welfare.

In Sweden, people with ID and/or autism spectrum disorder (ASD) may apply for service and support to manage their daily lives. This support is regulated in the Act Concerning Support and Service for Persons with Certain Functional Impairments (Swedish abbreviation: LSS) [15]. The support consists of ten different measures, whereof eight are available for adults: counselling, personal assistance, companion service, contact person, relief service in the home (for informal caregivers), short-time stay away from home (to relieve informal caregivers), special housing, and occupation at daily activities center. With the exception of counselling, which is provided by the county, all services are provided by the municipality and recorded in the so-called LSS-register.

The Swedish Prescribed Drug Register contains information on all dispensed prescribed drugs, recorded by their anatomical therapeutic chemical (ATC) classification code. Registration is done at the time of purchase, i.e., drugs that are prescribed but never dispensed are not included.

The Swedish National Patient Register (NPR) contains information on all inpatient and outpatient specialist care in Sweden. For each health care visit, one primary and up to 21 secondary diagnoses are recorded according to the International Statistical Classification of Diseases and Related Health Problems 10th Revision (ICD-10). Secondary diagnoses are to be recorded only if they are of relevance to the treatment of the primary diagnosis. Registration is done at discharge, i.e., ongoing hospitalizations are not included.

\section{Study population}

We used the LSS-register to identify all people aged at least 55 years and alive at the end of 2012, and with at least one measure of support during this year. This resulted in a cohort of 7936 people with ID, whereof 3609 (45\%) were women and 4327 (55\%) were men. In the analyses of demographic variables, we only included those with complete demographic data (see below), resulting in a subcohort comprising 1151 people with ID (554 women and 597 men). The mean age on December 31, 2012 was 64 years (range 55-96) in the total cohort and 63 years (range 55-88) in the subcohort with complete demographic data.

\section{Antipsychotics}

Data on prescription of antipsychotics were obtained from the Swedish Prescribed Drug Register. Based on the assumption that prescriptions of antipsychotics in this population are primarily symptomatic and off-label, frequently with an objective to calm the patients and reduce behavioral problems, we chose to categorize antipsychotics as sedating [chlorpromazine (N05AA01), levomepromazine (N05AA02), thioridazine (N05AC02), clozapine (N05AH02), olanzapine (N05AH03), quetiapine (N05AH04), chlorprothixene (N05AF03), melperone (N05AD03), perfenazine (N05AB03), zuclopenthixol (N05AF05), and dixyrazine (N05AB01)] and less-sedating [fluphenazine (N05AB02), haloperiodol (N05AD01), flupentixol (N05AF01), sertindole (N05AE03), ziprasidone (N05AE04), risperidone (N05AX08), paliperidone (N05AX13), and aripiprazole (N05AX12)] antipsychotics.

\section{Demographic data}

When considering demographic data, we included severity of ID, presence of behavior impairment (as determined by the fourth digit of the F7-dignosis), sex, year of birth (dichotomized at the median for the total cohort, i.e., January 1,1950 ), and residing in special housing for people with ID and/or ASD at least 1 year during the study period. Data on special housing were collected from the LSS register, whereas information on ID severity and presence of impairment of behavior was collected from the Swedish NPR. Using data on diagnoses during 2002-2012, we identified those who had at least one specified (i.e., not "other" or "unknown" ID) diagnosis of ID (F7) during this period. These were categorized as having severe/profound $(n=255)$, moderate $(n=285)$, or mild $(n=611)$ ID. For each diagnosis of ID, it is possible for the physician to indicate whether the diagnosis is associated with impairment of behavior. Among those with specified ID diagnosis, we identified 361 people (160 women and 201 men) with and 790 (394 women and 396 men) without impairment of behavior.

\section{Diagnostic profiles}

We collected information on all diagnoses (i.e., both primary and secondary) in ICD-10 chapters I-XVII recorded in the NPR during the study period (2006-2012), on ICD-10 block level. Diagnoses that were present among at least five people 
with and without prescription of sedating and less-sedating antipsychotics, respectively, were assessed as outcomes, i.e., investigated for associations with the prescription of antipsychotics. Exclusion of diagnoses with less registrations was done partly due to considerations concerning statistical power and partly to avoid presenting data that might make it possible to identify a specific individual.

Using data from 2002 to 2012, we also identified people with diagnosis of at least one disorder that is an indicator of prescription of antipsychotics schizophrenia (F20), bipolar affective disorder (F31), restlessness, and agitation (R45.1), Tourette syndrome (F95.2), generalized nonpsychotic anxiety (F41.1), or schizoaffective disorders (F25) $(n=240$, $21 \%$ ). To simplify reading, we will henceforth refer to these as on-label indications.

\section{Statistics}

Demographic variables were analyzed in multivariate models including all demographic variables and the on-label indications simultaneously. Analyses were performed on the subcohort with complete demographic data $(n=1151)$. Diagnoses were analyzed using multivariate models including all selected diagnoses (i.e., present in at least five people with and five people without prescription) as well as sex and year of birth (dichotomized at the median). In these analyses, all 7936 people in the cohort were included.

Analyses were performed using generalized linear models (GLMs), estimating relative risks (RRs) with 95\% confidence intervals (CIs). All analyses were performed in IBM SPSS Statistics 25.0. Two-sided $p$ values lower than 0.05 were considered statistically significant.

\section{Results}

In the total cohort (i.e., among the 7936 people with ID), at least one prescription of antipsychotics was found for 3111 (39\%) people. Of these, 1385 (45\%) had prescription of sedating antipsychotics only, 1047 (34\%) of less-sedating antipsychotics only, and 679 (22\%) of both sedating and less-sedating antipsychotics. Among the 1151 people with complete demographic data, at least one prescription of antipsychotics was found for 808 (70\%) people. Of these, $363(45 \%)$ had prescription of sedating antipsychotics only, $217(27 \%)$ of less-sedating antipsychotics only, and 228 $(28 \%)$ of both sedating and less-sedating antipsychotics.

In the subcohort of people with complete demographic data, prescription of at least one sedating antipsychotic during the study period was associated with severe/profound ID, behavior impairment, and living in special housing at least 1 year during the study period (Table 1). For less-sedating antipsychotics, the only statistically significant association was found for behavior impairment, which was associated with an increased risk of prescription.

Using data from all 7936 people in the total cohort, nine diagnoses were positively associated with prescription of sedating antipsychotics (i.e., those with a diagnosis more likely to have a prescription) in multivariate analyses adjusted for sex and year of birth (Table 2). The largest effects (i.e., highest RRs) were found for schizophrenia, schizotypal, and delusional disorders (F20-F29 in ICD-10), mental retardation (i.e., ID; F70-F79), and disorders of psychological development (F80-F89). Negative associations (i.e., those with a diagnosis less likely to have a prescription) were found for 12 diagnoses. The largest effects (i.e., lowest RRs) were found for inflammatory polyarthropathies (M05-M14), other disorders of the nervous system (G90-G99), and chromosomal abnormalities, not elsewhere classified (Q90-Q99).

In the analyses of less-sedating antipsychotics, eight diagnoses were positively associated with prescription, with the largest effects found for schizophrenia, schizotypal, and delusional disorders (F20-F29 in ICD-10), mental retardation (i.e., ID; F70-F79), and organic, including symptomatic, mental disorders (F00-F09). A negative association was found only for disorders of choroid and retina (H30-H36).

\section{Discussion}

We found a high prescription of antipsychotics among older people with ID and behavior impairment, even when adjusting for on-label indications, as well as sex, year of birth, and severity of ID.

Before discussing the results of the present study, some methodological issues need to be addressed. The major strength of the study is the use of national registers to identify the cohorts and collect data on diagnoses and drug prescriptions. When assessing potential effects of demographic factors on the prescription of antipsychotics, we adjusted the analyses for diagnoses that are indicators for prescription of such drugs, i.e., where prescription is to be expected. The validity and reliability of psychiatric disorders are generally high in the Swedish NPR [16-18]. Even so, the restriction of data availability to specialist care may have caused us to miss persons with on-label indications made in primary care only. However, in the clinical experience of the authors, it is uncommon that these diagnoses are made in primary care only, with no follow-up in specialist care. Another potential weakness is that, due to the left truncation of data, we may have failed to identify persons with on-label indications diagnosed before the start of the study period. However, we see no reason that the fraction of people with diagnosis only from primary care or diagnosis made before the start of the 
Table 1 People with and without at least one prescription of sedating and less-sedating antipsychotics $(n=1151)$, during the study period (2006-2012)

\begin{tabular}{|c|c|c|c|c|c|}
\hline & \multicolumn{2}{|l|}{ Sedating } & \multicolumn{3}{|l|}{ Less-sedating } \\
\hline & No & Yes & No & & Yes \\
\hline \multicolumn{6}{|l|}{ Sex } \\
\hline Women; $n(\%)$ & $286(52)$ & $268(48)$ & $332(60)$ & & $222(40)$ \\
\hline Men; $n(\%)$ & $274(46)$ & $323(54)$ & $374(63)$ & & $223(37)$ \\
\hline \multicolumn{6}{|l|}{ Men vs women } \\
\hline RR $(95 \%$ CI $)$ & $1.11(0.99-1.23)$ & & $0.93(0.80-1.07)$ & & \\
\hline$p$ & 0.071 & & 0.310 & & \\
\hline \multicolumn{6}{|l|}{ Year of birth } \\
\hline$-1949 ; n(\%)$ & $273(51)$ & $266(49)$ & $336(62)$ & & $203(38)$ \\
\hline $1950-; n(\%)$ & 287 (47) & $325(53)$ & $370(60)$ & & $242(40)$ \\
\hline \multicolumn{6}{|l|}{ After vs before 1950} \\
\hline $\mathrm{RR}(95 \% \mathrm{CI})$ & $1.09(0.98-1.22)$ & & $1.06(0.92-1.23)$ & & \\
\hline $\mathrm{p}$ & 0.106 & & 0.422 & & \\
\hline \multicolumn{6}{|l|}{ Severity of ID } \\
\hline Mild; $n(\%)$ & $329(54)$ & $282(46)$ & $378(62)$ & & $233(38)$ \\
\hline Moderate; $n(\%)$ & $118(41)$ & $167(59)$ & $166(58)$ & & $119(42)$ \\
\hline Severe/profound; $n(\%)$ & $113(44)$ & $142(56)$ & $162(64)$ & & $93(36)$ \\
\hline \multicolumn{6}{|l|}{ Moderate vs mild ID } \\
\hline RR $(95 \% \mathrm{CI})$ & $1.13(0.99-1.28)$ & & $0.99(0.83-1.17)$ & & \\
\hline $\mathrm{p}$ & 0.063 & & 0.968 & & \\
\hline \multicolumn{6}{|c|}{ Severe/profound vs mild ID } \\
\hline $\mathrm{RR}(95 \% \mathrm{CI})$ & 1.17 (1.02-1.35) & & $0.91(0.75-1.10)$ & & \\
\hline $\mathrm{p}$ & 0.029 & & 0.325 & & \\
\hline \multicolumn{6}{|l|}{ Behavior impairment } \\
\hline Without; $n(\%)$ & $438(55)$ & $352(45)$ & $526(67)$ & & $264(33)$ \\
\hline With; $n(\%)$ & $122(34)$ & $239(66)$ & $180(50)$ & & $181(50)$ \\
\hline \multicolumn{6}{|l|}{ With vs without } \\
\hline RR $(95 \%$ CI $)$ & $1.34(1.20-1.50)$ & & $1.42(1.22-1.65)$ & & \\
\hline $\mathrm{p}$ & $<0.001$ & & $<0.001$ & & \\
\hline \multicolumn{6}{|l|}{ Living in special housing } \\
\hline Never & $107(61)$ & $68(39)$ & $118(67)$ & $57(33)$ & \\
\hline At least 1 year & $453(46)$ & $523(54)$ & $588(60)$ & $388(40)$ & \\
\hline \multicolumn{6}{|l|}{ At least 1 year vs never } \\
\hline $\mathrm{RR}(95 \% \mathrm{CI})$ & $1.25(1.04-1.50)$ & & $1.16(0.93-1.46)$ & & \\
\hline $\mathrm{p}$ & 0.019 & & 0.192 & & \\
\hline
\end{tabular}

Relative risks (RRs) with 95\% confidence intervals (CIs) and $p$ values from models including demographic variables as well as diagnoses that are indicators for prescription of antipsychotics are presented. Bold text marks statistically significant effects study should differ between, e.g., men or women, or people with and without behavior impairment. Thus, potential misclassification of on-label indications should not have played a major role in the present study.

The Swedish Prescribed Drug Register contains data on all dispensed prescribed drugs in the country, accounting for $84 \%$ of the total utilization [19]. As antipsychotics are not available for over-the-counter purchases, the coverage can be expected to be even higher for these drugs. However, drugs dispensed at hospital wards are not included in the register, and thus we may have failed to correctly classify people who received drugs at hospital but not by prescription. Nevertheless, we expect such misclassification to be unlikely considering the length of the study period.

As the Swedish Prescribed Drug Register includes only dispensed drugs, we have no information on prescriptions that were never filled (i.e., primary non-adherence). However, Swedish patients are included in an insurance system, a co-pay system, in which the care recipient has a highcost protection for non-institutional health care as well as for the purchase of pharmaceuticals within the pharmaceutical benefit program. Moreover, the studied group consists 
Table 2 Comorbidities and prescription of sedating and less-sedating antipsychotics, respectively

\begin{tabular}{|c|c|c|c|c|}
\hline & \multicolumn{2}{|l|}{ Sedating } & \multicolumn{2}{|l|}{ Less-sedating } \\
\hline & $\mathrm{RR}(95 \% \mathrm{CI})$ & $p$ & $\mathrm{RR}(95 \% \mathrm{CI})$ & $p$ \\
\hline A00-A09 intestinal infectious diseases & $0.68(0.47-0.98)$ & 0.040 & $0.91(0.63-1.32)$ & 0.625 \\
\hline A30-A49 other bacterial diseases & $0.99(0.81-1.20)$ & 0.895 & $0.93(0.74-1.17)$ & 0.538 \\
\hline B35-B49 mycoses & NI & & $1.24(0.87-1.75)$ & 0.230 \\
\hline B95-B98 bacterial, viral, and other infectious agents & $1.05(0.81-1.34)$ & 0.724 & $0.90(0.67-1.21)$ & 0.476 \\
\hline C15-C26 malignant neoplasms of digestive organs & $1.01(0.69-1.46)$ & 0.978 & $0.91(0.56-1.47)$ & 0.688 \\
\hline C43-C44 melanoma and other malignant neoplasms of skin & $0.84(0.65-1.09)$ & 0.191 & $1.06(0.82-1.37)$ & 0.664 \\
\hline C50-C50 malignant neoplasm of breast & $1.13(0.86-1.48)$ & 0.373 & $0.91(0.63-1.31)$ & 0.602 \\
\hline D10-D36 benign neoplasms & $0.75(0.53-1.05)$ & 0.095 & $0.79(0.50-1.23)$ & 0.299 \\
\hline D37-D48 neoplasms of uncertain or unknown behaviour & $1.12(0.80-1.56)$ & 0.521 & $0.99(0.66-1.47)$ & 0.950 \\
\hline D50-D53 nutritional anaemias & $0.72(0.54-0.97)$ & $\mathbf{0 . 0 3 0}$ & $1.02(0.78-1.35)$ & 0.870 \\
\hline D60-D64 aplastic and other anaemias & $1.04(0.85-1.27)$ & 0.706 & $0.94(0.74-1.19)$ & 0.594 \\
\hline E00-E07 disorders of thyroid gland & $0.97(0.79-1.18)$ & 0.740 & $0.94(0.75-1.18)$ & 0.593 \\
\hline E10-E14 diabetes mellitus & $1.03(0.91-1.17)$ & 0.629 & $1.25(1.09-1.44)$ & 0.001 \\
\hline E65-E68 obesity and other hyperalimentation & $0.83(0.59-1.17)$ & 0.288 & $0.66(0.43-1.01)$ & 0.056 \\
\hline E70-E90 metabolic disorders & $1.19(1.03-1.37)$ & 0.016 & $1.06(0.89-1.27)$ & 0.494 \\
\hline F00-F09 organic, including symptomatic, mental disorders & $1.23(1.07-1.41)$ & 0.003 & $1.55(1.35-1.78)$ & $<0.001$ \\
\hline F10-F19 mental and behavioral disorders due to psychoactive substance use & $0.91(0.73-1.14)$ & 0.423 & $0.77(0.58-1.04)$ & 0.092 \\
\hline F20-F29 schizophrenia, schizotypal, and delusional disorders & $2.17(1.99-2.36)$ & $<0.001$ & $1.81(1.60-2.04)$ & $<0.001$ \\
\hline F30-F39 mood [affective] disorders & $1.48(1.33-1.64)$ & $<0.001$ & $1.36(1.20-1.54)$ & $<0.001$ \\
\hline F40-F48 neurotic, stress-related and somatoform disorders & $1.21(1.07-1.37)$ & 0.002 & $1.30(1.12-1.51)$ & $<0.001$ \\
\hline $\begin{array}{l}\text { F50-F59 behavioral syndromes associated with physiological disturbances and physical } \\
\text { factors }\end{array}$ & NI & & $1.32(0.81-2.15)$ & 0.259 \\
\hline F60-F69 disorders of adult personality and behaviour & $1.17(0.94-1.46)$ & 0.153 & $1.00(0.73-1.38)$ & 0.976 \\
\hline F70-F79 mental retardation & $1.84(1.70-1.99)$ & $<0.001$ & $1.68(1.54-1.85)$ & $<0.001$ \\
\hline F80-F89 disorders of psychological development & $1.57(1.44-1.71)$ & $<0.001$ & $1.34(1.20-1.50)$ & $<0.001$ \\
\hline $\begin{array}{l}\text { F90-F98 behavioral and emotional disorders with onset usually occurring in childhood } \\
\text { and adolescence }\end{array}$ & $1.03(0.80-1.32)$ & 0.823 & $1.53(1.20-1.95)$ & 0.001 \\
\hline G20-G26 extrapyramidal and movement disorders & $1.020 .78-1.33)$ & 0.895 & $1.00(0.71-1.41)$ & 0.991 \\
\hline G40-G47 episodic and paroxysmal disorders & $0.90(0.82-0.99)$ & $\mathbf{0 . 0 3 7}$ & $0.92(0.82-1.03)$ & 0.141 \\
\hline G80-G83 cerebral palsy and other paralytic syndromes & $0.64(0.52-0.80)$ & $<0.001$ & $0.85(0.68-1.06)$ & 0.151 \\
\hline G90-G99 other disorders of the nervous system & $0.51(0.29-0.91)$ & 0.022 & $0.89(0.59-1.33)$ & 0.560 \\
\hline $\mathrm{H} 15-\mathrm{H} 22$ disorders of sclera, cornea, iris, and ciliary body & $0.82(0.57-1.18)$ & 0.287 & $1.10(0.79-1.54)$ & 0.571 \\
\hline $\mathrm{H} 25-\mathrm{H} 28$ disorders of lens & $0.86(0.75-0.99)$ & $\mathbf{0 . 0 3 4}$ & $1.03(0.89-1.19)$ & 0.684 \\
\hline H30-H36 disorders of choroid and retina & $1.00(0.80-1.26)$ & 0.983 & $0.61(0.47-0.80)$ & $<0.001$ \\
\hline H40-H42 glaucoma & $0.79(0.61-1.03)$ & 0.080 & $1.04(0.81-1.33)$ & 0.765 \\
\hline $\begin{array}{l}\text { H49-H52 disorders of ocular muscles, binocular movement, accommodation, and } \\
\text { refraction }\end{array}$ & $1.07(0.82-1.40)$ & 0.617 & $1.06(0.80-1.40)$ & 0.668 \\
\hline H53-H54 visual disturbances and blindness & $0.95(0.70-1.28)$ & 0.732 & $0.72(0.50-1.04)$ & 0.081 \\
\hline H90-H95 other disorders of ear & $1.06(0.81-1.40)$ & 0.656 & $0.83(0.55-1.25)$ & 0.375 \\
\hline I10-I15 hypertensive diseases & $0.63(0.54-0.72)$ & $<0.001$ & $0.87(0.75-1.01)$ & 0.072 \\
\hline I20-I25 ischaemic heart diseases & $1.06(0.88-1.27)$ & 0.525 & $0.88(0.70-1.12)$ & 0.308 \\
\hline I26-I28 pulmonary heart disease and diseases of pulmonary circulation & $1.25(0.94-1.65)$ & 0.125 & $0.92(0.59-1.43)$ & 0.706 \\
\hline I30-I52 other forms of heart disease & $0.90(0.78-1.03)$ & 0.136 & $0.99(0.84-1.17)$ & 0.938 \\
\hline I60-I69 cerebrovascular diseases & $1.09(0.92-1.30)$ & 0.314 & $0.96(0.78-1.18)$ & 0.682 \\
\hline I80-I89 diseases of veins, lymphatic vessels, and lymph nodes, not elsewhere classified & $0.85(0.64-1.12)$ & 0.242 & $\mathrm{NI}$ & \\
\hline I95-I99 other and unspecified disorders of the circulatory system & $0.97(0.69-1.39)$ & 0.888 & $1.34(0.92-1.94)$ & 0.387 \\
\hline J00-J06 acute upper respiratory infections & $0.96(0.76-1.22)$ & 0.739 & $1.12(0.88-1.42)$ & 0.590 \\
\hline J09-J18 influenza and pneumonia & $1.10(0.98-1.24)$ & 0.094 & $1.09(0.95-1.24)$ & 0.793 \\
\hline J20-J22 other acute lower respiratory infections & $0.68(0.49-0.95)$ & 0.025 & $1.28(0.98-1.68)$ & 0.455 \\
\hline
\end{tabular}


Table 2 (continued)

\begin{tabular}{|c|c|c|c|c|}
\hline & \multicolumn{2}{|l|}{ Sedating } & \multicolumn{2}{|l|}{ Less-sedating } \\
\hline & $\mathrm{RR}(95 \% \mathrm{CI})$ & $p$ & $\mathrm{RR}(95 \% \mathrm{CI})$ & $p$ \\
\hline J30-J39 other diseases of upper respiratory tract & $0.95(0.75-1.21)$ & 0.691 & $0.94(0.70-1.27)$ & 0.981 \\
\hline J40-J47 chronic lower respiratory diseases & $1.27(1.08-1.50)$ & 0.003 & $0.92(0.73-1.16)$ & 0.931 \\
\hline J60-J70 lung diseases due to external agents & $0.96(0.75-1.23)$ & 0.746 & $1.04(0.78-1.39)$ & 0.983 \\
\hline J90-J94 other diseases of pleura & $\mathrm{NI}$ & & $0.90(0.57-1.43)$ & 0.504 \\
\hline J95-J99 other diseases of the respiratory system & $1.25(0.92-1.68)$ & 0.151 & $0.83(0.54-1.27)$ & 0.429 \\
\hline K20-K31 diseases of oesophagus, stomach, and duodenum & $0.98(0.83-1.16)$ & 0.803 & $0.95(0.79-1.14)$ & 0.782 \\
\hline K40-K46 hernia & $0.87(0.73-1.03)$ & 0.103 & $0.98(0.81-1.17)$ & 0.959 \\
\hline K50-K52 noninfective enteritis and colitis & $0.65(0.47-0.91)$ & 0.012 & $1.12(0.83-1.50)$ & 0.705 \\
\hline K55-K63 other diseases of intestines & $1.07(0.95-1.20)$ & 0.267 & $1.00(0.86-1.15)$ & 0.393 \\
\hline K80-K87 disorders of gallbladder, biliary tract, and pancreas & $0.84(0.66-1.06)$ & 0.146 & $1.01(0.80-1.28)$ & 0.987 \\
\hline K90-K93 other diseases of the digestive system & $1.40(1.17-1.67)$ & $<0.001$ & $1.00(0.79-1.26)$ & 0.261 \\
\hline L00-L08 infections of the skin and subcutaneous tissue & $0.89(0.61-1.29)$ & 0.539 & $1.14(0.78-1.67)$ & 0.792 \\
\hline L40-L45 papulosquamous disorders & NI & & $1.25(0.72-2.14)$ & 0.664 \\
\hline L80-L99 other disorders of the skin and subcutaneous tissue & $0.65(0.46-0.92)$ & 0.016 & $0.95(0.67-1.36)$ & 0.381 \\
\hline M05-M14 inflammatory polyarthropathies & $0.51(0.30-0.87)$ & 0.014 & $1.01(0.72-1.42)$ & 0.819 \\
\hline M15-M19 arthrosis & $0.98(0.83-1.15)$ & 0.822 & $1.04(0.86-1.25)$ & 0.228 \\
\hline M20-M25 other joint disorders & $0.90(0.69-1.19)$ & 0.472 & $0.87(0.64-1.19)$ & 0.570 \\
\hline M50-M54 other dorsopathies & $0.88(0.61-1.25)$ & 0.464 & $1.00(0.70-1.43)$ & 0.573 \\
\hline M70-M79 other soft tissue disorders & $0.93(0.80-1.09)$ & 0.358 & $1.10(0.93-1.31)$ & 0.124 \\
\hline M80-M85 disorders of bone density and structure & $1.17(0.92-1.47)$ & 0.195 & $0.96(0.73-1.27)$ & 0.387 \\
\hline N10-N16 renal tubulo-interstitial diseases & $0.74(0.54-1.03)$ & 0.074 & $1.07(0.79-1.46)$ & 0.590 \\
\hline N17-N19 renal failure & $0.99(0.74-1.32)$ & 0.937 & $0.87(0.63-1.20)$ & 0.793 \\
\hline $\mathrm{N} 20-\mathrm{N} 23$ urolithiasis & $0.78(0.47-1.28)$ & 0.327 & $0.94(0.57-1.56)$ & 0.455 \\
\hline N30-N39 other diseases of urinary system & $1.00(0.89-1.12)$ & 0.940 & $0.92(0.79-1.06)$ & 0.981 \\
\hline N40-N51 diseases of male genital organs & $0.71(0.50-1.00)$ & 0.052 & $0.91(0.64-1.27)$ & 0.931 \\
\hline N80-N98 noninflammatory disorders of female genital tract & $0.67(0.43-1.05)$ & 0.082 & $0.88(0.57-1.36)$ & 0.983 \\
\hline Q90-Q99 chromosomal abnormalities, not elsewhere classified & $0.59(0.46-0.74)$ & $<0.001$ & $1.15(0.96-1.38)$ & 0.504 \\
\hline
\end{tabular}

Relative risks (RRs) with 95\% confidence intervals (CIs) and $p$ values from models including all diagnoses present in at least five people with and without each respective antipsychotic, and adjusted for demographic variables (sex, year of birth). Bold text marks statistically significant effects

NI not included due to too few observations NI

of individuals receiving special daily living service and support from the municipality, and may be assumed to be monitored with respect to drug adherence to a higher degree than people in the general population. This should be true also for secondary non-adherence (patients taking insufficient doses required to experience a therapeutic effect, missing doses or discontinuing therapy early). Thus, both types of non-adherence could be expected to be a lesser issue in this population than in other.

During the 6-year long study period, we found at least one prescription of antipsychotics for $70 \%$ of the study cohort. This is slightly higher than psychotropic prescription rates (59-61\%) in previous population cohort studies of older people with ID [20,21], but similar to the $68 \%$ found among inpatients with ID [12]. A potential explanation for this discrepancy is the use of a national register with high coverage in the present study.

We found no effect of sex or year of birth on the prescription of antipsychotics, which is in agreement with earlier studies [21], and also with data for the general population in regional Swedish data (Region Skåne chief pharmacist Naida Al-Omar, personal communication).

In line with previous research $[8,21]$, people with behavior impairment, and moderate and severe/profound ID were more likely to be prescribed antipsychotics. In the present study, this was seen even after taking into account diagnoses that are indicators for such prescription. Off-label prescription of antipsychotics is common among people with ID [12], and one interpretation of our findings is that it is even more common among those with behavior impairment and 
more severe ID. It has been suggested that the high rates of prescription of antipsychotics are due to the use to treat challenging behavior $[9-12,22]$. This in spite that there is not enough evidence to recommend any specific medication for such behavior [13], and the use of antipsychotics has been found not to be cost-effective [14]. However, it might also be argued that as manifestations of psychosis, bipolar disorders and other psychiatric disorders often are atypical and difficult to diagnose in persons with ID, challenging behaviors may be difficult to differentiate from psychotic symptoms. If so, "off-label" prescription may be the correct treatment for the indication even if a diagnosis has not been made. Still, a more person-centered approach would be to attempt to determine the cause of the challenging behavior. In a previous study, we found that older people with ID were less likely than their age-peers in the general population to be diagnosed with several types of pain [23]. In the same study, we found that even when a diagnosis of pain was made, prescription of pain medication differed between people with ID and the general population. It is not a stretch to imagine that undiagnosed or untreated pain may lead to challenging behavior, especially among people with difficulties both in understanding and communicating their symptoms.

In register-based studies, findings regarding differences in comorbidity spectrum between patients on sedating and less-sedating antipsychotics, respectively, need to be interpreted with caution. Even so, it is interesting to note that we did not find any heightened risk of either cardiovascular disease or diabetes mellitus associated with prescription of sedative antipsychotics, such as olanzapine, which has been suggested previously [24]. One potential explanation for this could be that a reason why some patients received sedative antipsychotics is that they were comparatively more agitated, physically active, and less sedentary. This is a finding worth further investigation.

To decrease the prescription of antipsychotics as off-label to people diagnosed with ID there seems to be a need for a general health care reinforcement including the implementation of guideline recommendations $[25,26]$ and for the team of health professionals in the patient's network to adhere, facilitate, and enforce the recommendations implemented.

\section{Conclusions}

Psychiatric disorders are difficult to diagnose in ID, with symptoms being masked by manifestations of behavioral impairment. Hence, it is reasonable to assume that at least part of the "off-label" prescriptions of antipsychotics is done for indications that are present but undiagnosed, and that the high rates of antipsychotic prescriptions found for older people with ID might be warranted by a presence of "hidden indicator symptoms". In addition to the diagnostic difficulties, there is also an administrative pressure with psychiatrists having to compensate for lack of staff needed to handle the special needs of individuals with ID. Even so, this is a patient category where proper diagnosis may demand extra time, and where the clinical management of psychiatric disorders is more time consuming than in non-ID populations. Thus, it is important to spread information about diagnosing and treating psychiatric disorders in people with ID among health care providers. Also, to encourage regular medication revisions, and most importantly to develop and improve the ability of health care professionals to properly diagnose psychiatric illness in this population.

There are strong associations between prescription of sedating antipsychotics and several diagnoses that are not indicators for antipsychotics. Considering the inherent vulnerability of the population with ID and the large range of potential side effects of antipsychotics, it is particularly important that prescription of these drugs always follow a thorough assessment of the needs, and consideration of possible alternative treatments.

Acknowledgements This work was funded by Forte, the Swedish Research Council for Health, Working Life and Welfare no. 2014-4753.

\section{Compliance with ethical standards}

Conflict of interest The authors declare that they have no conflict of interest.

Ethical standards The study was approved by the Regional Ethical Review Board (Lund) and have therefore been performed in accordance with the ethical standards laid down in the 1964 Declaration of Helsinki and its later amendments. The manuscript does not contain clinical studies or patient data.

Open Access This article is distributed under the terms of the Creative Commons Attribution 4.0 International License (http://creativeco mmons.org/licenses/by/4.0/), which permits unrestricted use, distribution, and reproduction in any medium, provided you give appropriate credit to the original author(s) and the source, provide a link to the Creative Commons license, and indicate if changes were made.

\section{References}

1. American Geriatrics Society (2015) American Geriatrics Society 2015 updated beers criteria for potentially inappropriate medication use in older adults. J Am Geriatr Soc 63(11):2227-2246. https ://doi.org/10.1111/jgs.13702

2. Both PH, Sonneveld DS, Beumer S, Bierma-Zeinstra SMA, MaesFesten DAM (2017) Movement disorders in adults with intellectual disability and behavioral problems associated with use of antipsychotics? J Clin Psychopharmacol 37(3):385-387. https:// doi.org/10.1097/JCP.0000000000000705

3. National Board of Health and Welfare (2010) Indicators of good drug therapy in the elderly [In Swedish: Indikatorer för god 
läkemedelsterapi hos äldre]. Swedish National Board of Health and Welfare (Socialstyrelsen), Stockholm

4. Scheifes A, Walraven S, Stolker JJ, Nijman HL, Egberts TC, Heerdink ER (2016) Adverse events and the relation with quality of life in adults with intellectual disability and challenging behaviour using psychotropic drugs. Res Dev Disabil 49-50:13-21. https://doi.org/10.1016/j.ridd.2015.11.017

5. Chutka DS, Takahashi PY, Hoel RW (2004) Inappropriate medications for elderly patients. Mayo Clin Proc 79(1):122-139. https:// doi.org/10.4065/79.1.122

6. Aman H, Naeem F, Farooq S, Ayub M (2016) Prevalence of nonaffective psychosis in intellectually disabled clients: systematic review and meta-analysis. Psychiatr Genet 26(4):145-155. https ://doi.org/10.1097/ypg.0000000000000137

7. Hermans H, Beekman AT, Evenhuis HM (2014) Comparison of anxiety as reported by older people with intellectual disabilities and by older people with normal intelligence. Am J Geriatr Psychiatry 22(12):1391-1398. https://doi.org/10.1016/j. jagp.2013.04.017

8. Sheehan R, Hassiotis A, Walters K, Osborn D, Strydom A, Horsfall L (2015) Mental illness, challenging behaviour, and psychotropic drug prescribing in people with intellectual disability: UK population based cohort study. BMJ 351:h4326. https://doi. org/10.1136/bmj.h4326

9. Doan TN, Lennox NG, Taylor-Gomez M, Ware RS (2013) Medication use among Australian adults with intellectual disability in primary healthcare settings: a cross-sectional study. J Intellect Dev Disabil 38(2):177-181. https://doi.org/10.3109/13668 250.2013.778968

10. Deb S, Unwin G, Deb T (2015) Characteristics and the trajectory of psychotropic medication use in general and antipsychotics in particular among adults with an intellectual disability who exhibit aggressive behaviour. J Intellect Disabil Res 59(1):11-25. https:// doi.org/10.1111/jir.12119

11. Tsiouris JA, Kim SY, Brown WT, Pettinger J, Cohen IL (2013) Prevalence of psychotropic drug use in adults with intellectual disability: positive and negative findings from a large scale study. $\mathrm{J}$ Autism Dev Disord 43(3):719-731. https://doi.org/10.1007/s1080 3-012-1617-6

12. Haw C, Stubbs J (2005) A survey of off-label prescribing for inpatients with mild intellectual disability and mental illness. J Intellect Disabil Res 49(Pt 11):858-864. https://doi.org/10.111 1/j.1365-2788.2005.00723.x

13. Deb S, Sohanpal SK, Soni R, Lenotre L, Unwin G (2007) The effectiveness of antipsychotic medication in the management of behaviour problems in adults with intellectual disabilities. J Intellect Disabil Res 51(Pt 10):766-777. https://doi.org/10.111 1/j.1365-2788.2007.00950.x

14. Romeo R, Knapp M, Tyrer P, Crawford M, Oliver-Africano P (2009) The treatment of challenging behaviour in intellectual disabilities: cost-effectiveness analysis. J Intellect Disabil Res 53(7):633-643. https://doi.org/10.1111/j.1365-2788.2009.01180.x

15. SFS1993:387 (1993) Act Concerning support and service for persons with certain functional impairments [In Swedish: Lag om stöd och service till vissa funktionshindrade]. Ministry of Health and Social Affairs, Stockholm

16. Ruck C, Larsson KJ, Lind K, Perez-Vigil A, Isomura K, Sariaslan A, Lichtenstein P, Mataix-Cols D (2015) Validity and reliability of chronic tic disorder and obsessive-compulsive disorder diagnoses in the Swedish National Patient Register. BMJ Open 5(6):e007520. https://doi.org/10.1136/bmjopen-2014-007520

17. Sellgren C, Landen M, Lichtenstein P, Hultman CM, Langstrom N (2011) Validity of bipolar disorder hospital discharge diagnoses: file review and multiple register linkage in Sweden. Acta Psychiatr Scand 124(6):447-453. https://doi.org/10.111 1/j.1600-0447.2011.01747.x

18. Allebeck P (2009) The use of population based registers in psychiatric research. Acta Psychiatr Scand 120(5):386-391. https:// doi.org/10.1111/j.1600-0447.2009.01474.x

19. Wettermark B, Hammar N, Fored CM, Leimanis A, Otterblad Olausson P, Bergman U, Persson I, Sundstrom A, Westerholm B, Rosen M (2007) The new Swedish Prescribed Drug Register-opportunities for pharmacoepidemiological research and experience from the first six months. Pharmacoepidemiol Drug Saf 16(7):726-735. https://doi.org/10.1002/pds.1294

20. O'Dwyer C, McCallion P, Burke E, Carroll R, O'Dwyer M, McCarron M (2018) Prevalence and associated factors of problem behaviours among older adults with intellectual disabilities in Ireland. Res Dev Disabil 80:192-204. https://doi.org/10.1016/j. ridd.2018.05.007

21. O’Dwyer M, Peklar J, Mulryan N, McCallion P, McCarron M, Henman MC (2017) Prevalence, patterns and factors associated with psychotropic use in older adults with intellectual disabilities in Ireland. J Intellect Disabil Res 61(10):969-983. https://doi. org/10.1111/jir.12391

22. Deb S (2007) The role of medication in the management of behaviour problems in people with learning disabilities. Adv Ment Health Learn Disabil 1(2):26-31. https://doi.org/10.1108/17530 180200700017 doi

23. Axmon A, Ahlström G, Westergren H (2018) Pain and pain medication among older people with intellectual disabilities in comparison with the general population. Healthcare 6(2):67. https:// doi.org/10.3390/healthcare6020067

24. Lublin H, Eberhard J, Levander S (2005) Current therapy issues and unmet clinical needs in the treatment of schizophrenia: a review of the new generation antipsychotics. Int Clin Psychopharmacol 20(4):183-198

25. Ramerman L, Hoekstra PJ, de Kuijper G (2018) Exploring barriers and facilitators in the implementation and use of guideline recommendations on antipsychotic drug prescriptions for people with intellectual disability. J Appl Res Intellect Disabil JARID. https://doi.org/10.1111/jar.12461

26. Royal College of Psychiatrist (2017) Use of licensed medicines for unlicensed applications in psychiatric practice. College Report, Royal College of Psychiatrists, London 